\title{
Mapeamento da organização dos fluxos de acesso aos serviços de saúde: Revisão Integrativa
}

\author{
Mapping of the organization of access flows to health services: Integrative Review \\ Mapeo de la organización de los flujos de acceso a los servicios de salud: Revisión Integrativa
}

Recebido: 09/12/2021 | Revisado: 17/12/2021 | Aceito: 22/12/2021 | Publicado: 04/01/2022

Rachel Saraiva Nogueira

ORCID: https://orcid.org/0000-0001-7860-4865 Universidade Estadual do Ceará, Brasil

E-mail: rachelsaraiva00@ gmail.com

Dina Mara Formiga da Silva

ORCID: https://orcid.org/0000-0002-5387-7194 Universidade Estadual do Ceará, Brasil E-mail: dinamara_silva@ hotmail.com

Maria Salete Bessa Jorge

ORCID: https://orcid.org/0000-0001-6461-3015 Universidade Estadual do Ceará, Brasi E-mail: maria.salete.jorge@gmail.com

\begin{abstract}
Resumo
Objetivo: Analisar o fluxo da regulação sanitária através do processo de emissão integral e encaminhamento definido da contrarreferência de forma a garantir a efetividade do sistema regional de saúde, a consecução do princípio da longitudinalidade do cuidado e o fortalecimento da rede de atenção à saúde. Método: Trata-se de uma Revisão Integrativa, onde seguiu-se as seis fases de Mendes, Silveira e Galvão. Resultados: 10 estudos inclusos nesta revisão que foram publicados e/ou disponibilizados no período de 1999 a 2020. Foi construído um formulário para a extração e o gerenciamento das informações. As informações foram organizadas por meio de um quadro com a descrição dos seguintes aspectos: ano de publicação, autoria, periódico/instituição, título, país do estudo e tipo de publicação. Os estudos sobre a temática abrangeram estudos de abordagem quantitativa, qualitativa ou qualiquantitativos, e com objetos de estudo semelhantes sobre a organização dos fluxos de acesso aos serviços de saúde. Conclusão: Os estudos demonstram que a acessibilidade é um desafio ubíquo e deficiências na porta de entrada prejudicam a consecução dos demais atributos essenciais da Atenção Primária à Saúde.
\end{abstract}

Palavras-chave: Serviços de saúde; Redes de Atenção; Referência; Contrarreferência.

\begin{abstract}
Objective: To analyze the flow of health regulation through the process of integral emission and defined referral of the counter-reference in order to ensure the effectiveness of the regional health system, the achievement of the principle of longitudinality of care and the strengthening of the health care network. Method: This is an Integrative Review, which followed the six phases of Mendes, Silveira and Galvão. Results: 10 studies included in this review that were published and/or made available from 1999 to 2020. A form has been constructed for the extraction and management of information. The information was organized through a table describing the following aspects: year of publication, authorship, journal/institution, title, country of study and type of publication. The studies on the subject covered studies of quantitative, qualitative or qualitative approach, and with similar objects of study on the organization of access flows to health services. Conclusion: The studies show that accessibility is a ubiquitous challenge and deficiencies in the entrance door hinder the achievement of the other essential attributes of Primary Health Care.
\end{abstract}

Keywords: Health services; Attention Networks; Reference; Counter-reference.

\section{Resumen}

Objetivo: Analizar el flujo de regulación sanitaria a través del proceso de emisión integral y derivación definida de la contrarreferencia con el fin de asegurar la efectividad del sistema regional de salud, el logro del principio de longitudinalidad de la atención y el fortalecimiento de la red asistencial. Método: Se trata de una Revisión Integradora, que siguió las seis fases de Mendes, Silveira y Galvão. Resultados: 10 estudios incluidos en esta revisión que se publicaron y/o pusieron a disposición entre 1999 y 2020. Se ha construido un formulario para la extracción y gestión de la información. La información se organizó a través de una tabla que describe los siguientes aspectos: año de publicación, autoría, revista/institución, título, país de estudio y tipo de publicación. Los estudios sobre el tema abarcaron estudios de enfoque cuantitativo, cualitativo o cualitativo, y con objetos de estudio similares sobre la organización de los flujos de acceso a los servicios de salud. Conclusión: Los estudios muestran que la accesibilidad 
es un reto ubicuo y las deficiencias en la puerta de entrada dificultan la consecución de los otros atributos esenciales de la Atención Primaria de Salud.

Palabras clave: Servicios de salud; Redes de Atención; Referencia; Contrarreferencia.

\section{Introdução}

O princípio da regionalização, para sua efetividade, depende de outro princípio fundamental: o das redes de atenção à saúde que são definidas como arranjos organizativos de ações e serviços de saúde, de diferentes densidades tecnológicas, que integradas por meio de sistemas técnico, logístico e de gestão, buscam garantir a integralidade do cuidado. Para que a regionalização no SUS seja efetiva por meio dos pontos integrantes das redes de atenção à saúde o sistema de referência e contrarreferência é, portanto, imprescindível (Ministério da Saúde, 2010).

Esse quadro da ausência de condições logísticas para operacionalizar e consequentemente baixa tradição no SUS quanto à função contrarreferência é um fator de perpetuação do agravo ainda não superado que é a fragmentação das ações e serviços de saúde no seu componente sistêmico. Nesse sentido, observa que diferentes situações se manifestam nas relações entre a Atenção Primária à Saúde -APS e a Atenção Ambulatorial Especializada- AAE: o generalista não conhece o especialista a quem refere a pessoa usuária, e o especialista não conhece o generalista a quem a contrarrefere (quando o faz); o generalista e o especialista nunca partilharam atividades clínicas ou educacionais; o especialista não tem as informações adequadas do generalista ao receber a pessoa encaminhada; o generalista não recebe as orientações do especialista ao ter de volta a pessoa; a pessoa se queixa de que o especialista parecia não saber porque ela estava ali com ele; o especialista não resolve o problema para o qual o generalista encaminhou; o especialista se apossa definitivamente da pessoa na atenção especializada (efeito velcro) (Mendes, 2019).

Para superação dessa realidade, pretende-se efetivar o fluxo da regulação sanitária através do processo de emissão integral e encaminhamento definido da contrarreferência de forma a garantir a efetividade do sistema regional de saúde, a consecução do princípio da longitudinalidade do cuidado e o fortalecimento da rede de atenção à saúde. Então, a conectividade é um preditor crítico de sucesso da coordenação do cuidado entre os generalistas e os especialistas e significa que os profissionais de saúde envolvidos dispõem da informação que necessitam e de um sistema de comunicação fluido para prestar os cuidados adequados.

De um lado, os generalistas devem estar seguros de que os especialistas sabem as razões das referências e tenham as informações necessárias para que possam desempenhar suas funções (referência); de outro, os especialistas devem prover as informações de volta que respondam às necessidades e às expectativas dos generalistas (contrarreferência). Isso em um ambiente em que os profissionais mantenham as pessoas usuárias informadas e confiantes de que eles estão comunicando entre si, em benefício dessas pessoas.

Esse estado de coisas precisa ser superado, sob pena da ineficiência técnica assistencial de toda uma rede de atenção especializada, cujo sentido da regulação fica comprometido a partir da função referência sem a contraprestação da função contrarreferência. Nesse sentido, esse estudo visa apresentar no contexto da região de saúde, a função contrarreferência de forma a subsidiar os serviços de origem da rede municipal consorciada no segmento da atenção à saúde da população referenciada.

Nesse sentido, este estudo tem como objetivo analisar as evidências científicas sobre o fluxo de acesso aos serviços de saúde com encaminhamentos (Referência) e resposta (Contrarreferência) garantindo a continuidade do cuidado. 


\section{Metodologia}

Trata-se de uma Revisão Integrativa, onde foram seguiu-se as seis etapas, a saber: 1) definição do tema e elaboração da pergunta de pesquisa; 2) critérios de elegibilidade, inclusão e exclusão dos estudos; 3) levantamento dos estudos selecionados em formato de tabelas, considerando todas as características em comum; 4) categorização e análise crítica dos achados, identificando diferenças e conflitos; 5) interpretação dos resultados e 6) apresentação dos resultados, incluindo análise crítica dos achados e síntese da revisão (Souza et al., 2010).

Para adequação às demais bases de dados e plataformas foram também utilizados os Descritores em Ciências da Saúde (DeCs) para as bases em português e para as bases de dados que utilizam o idioma inglês foram utilizados os descritores do Medical Subject Headings (MeSH). Junto aos descritores foram empregados os termos boolenos: AND, OR e NOT para compor as chaves de busca a serem utilizadas para buscas nas bases de dados.

As referências listadas nos estudos encontrados também foram pesquisadas, visando identificar documentos adicionais para inserção potencial. Dos estudos encontrados, foram incluídos 2 (dois) estudos nos idiomas inglês e 8 (oito) estudos em português; com abordagem quantitativa, qualitativa e quantiqualitativa; estudos primários; revisões sistemáticas, metanálises e/ou metasínteses.

\section{Resultados}

Um total de 406 referências foram inicialmente encontradas nas quatro bases de dados eletrônicas. Após a remoção dos artigos duplicados, os títulos e os resumos dos 391 artigos foram avaliados, e 10 estudos foram considerados potencialmente relevantes para leitura integral. Eles foram lidos e submetidos aos critérios de elegibilidade, e destes, 20 foram excluídos (por serem dissertações, teses ou capítulos de livro; por não abordarem assuntos referentes à acesso aos serviços de saúde, fluxos, referência e contrarreferência).

Percebeu-se que dez estudos foram considerados na presente revisão. A Figura 1 mostra o fluxograma de identificação, escaneamento e processo de inclusão adaptado do Preferred Reporting Items for Systematic Review and MetaAnalyses (PRISMA). 
Figura 1 - fluxograma de identificação, escaneamento e processo de inclusão adaptado do Preferred Reporting Items for Systematic Review and Meta-Analyses (PRISMA).
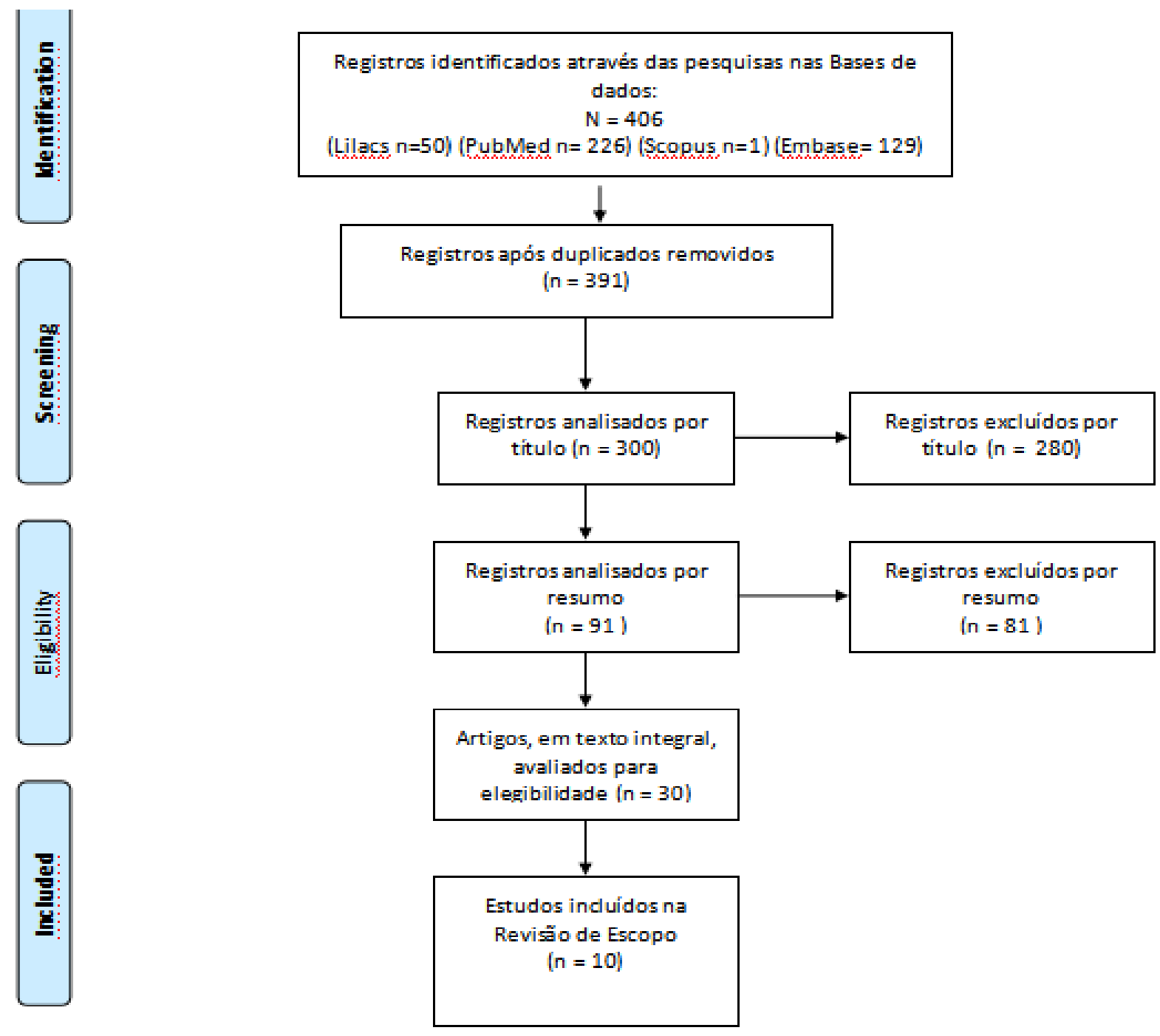

Fonte: Autores (2021).

As informações foram organizadas por meio de um quadro com a descrição dos seguintes aspectos: ano de publicação, autoria, periódico/instituição, título, país do estudo e tipo de publicação, conforme Quadro 2 (abaixo): 


\begin{tabular}{|c|c|c|c|c|c|}
\hline ANO & AUTOR & $\begin{array}{l}\text { PERIÓDICOI } \\
\text { INSTITUIÇÃO }\end{array}$ & TÍTULO & $\begin{array}{l}\text { PAÍS DO } \\
\text { ESTUDO }\end{array}$ & $\begin{array}{c}\text { TIPO DE } \\
\text { PUBLICAÇÃO }\end{array}$ \\
\hline 2017 & Hartxeit et al & $\begin{array}{l}\text { BMC Health Services } \\
\text { Research }\end{array}$ & $\begin{array}{c}\text { Indicadores de qualidade para o Processo de referência } \\
\text { da Saúde Mental primária à especializada do cuidado: } \\
\text { um estudo exploratório de acordo com método de } \\
\text { adequação }\end{array}$ & NORUEGA & ARTIGO \\
\hline 2018 & $\begin{array}{l}\text { Greenwoed-Lee et } \\
\text { al }\end{array}$ & $\begin{array}{l}\text { BMC Health Services } \\
\text { Research }\end{array}$ & $\begin{array}{c}\text { Uma categorização de problemas e soluções para } \\
\text { melhorar os encaminhamentos de pacientes do primário } \\
\text { para cuidados especiais }\end{array}$ & CANADÁ & ARTIGO \\
\hline 2020 & Katz et al & Ciência e Saúde Coletiva & $\begin{array}{c}\text { Acesso e regulação ao cuidado especializado no Rio } \\
\text { Grande do Sul: A estratégia RegulaSuS do Telesaudde - } \\
\text { UFRGS }\end{array}$ & BRASIL & ARTIGO \\
\hline 2018 & Lewis et al & $\begin{array}{c}\text { BMC Health Services } \\
\text { Research }\end{array}$ & $\begin{array}{c}\text { Reduzindo o tempo de espera do encaminhamento de } \\
\text { primeira visita para serviços ambulatoriais pode contribuir } \\
\text { para melhores resultados de saúde: uma revisão } \\
\text { sistemática }\end{array}$ & AUSTRÁLIA & ARTIGO \\
\hline 2013 & Erdmann et al & $\begin{array}{l}\text { Revista Latino-Americana } \\
\text { de Enfermagem }\end{array}$ & $\begin{array}{l}\text { A Atenção Secundária em Saúde: Melhores práticas na } \\
\text { rede de Serviços }\end{array}$ & BRASIL & ARTIGO \\
\hline 2014 & Xazquegetal et al & Ciência e Saúde Coletiva & $\begin{array}{l}\text { Referência e Contrarreferência na Atenção Secundária } \\
\text { em Odontologia em Campinas SP, Brasil }\end{array}$ & BRASIL & ARTIGO \\
\hline 1999 & Juliani et al & $\begin{array}{l}\text { Revista da Escola de } \\
\text { Enfermagem da USP }\end{array}$ & $\begin{array}{c}\text { Organização do Sistema de Referência e } \\
\text { Contrarreferência no Contexto do Sistema Único de } \\
\text { Saúde: a percepção dos Enfermeiros }\end{array}$ & BRASIL & ARTIGO \\
\hline 2009 & Einkelsztein et al & $\begin{array}{l}\text { Rhysis: Revista de Saúde } \\
\text { Coletiva }\end{array}$ & $\begin{array}{c}\text { Encaminhamentos da Atenção primária para avaliação } \\
\text { neurológica em Porto Alegre, Brasil }\end{array}$ & BRASIL & ARTIGO \\
\hline 2016 & Pereira et al & $\begin{array}{l}\text { Rhysis: Revista de Saúde } \\
\text { Coletiva }\end{array}$ & $\begin{array}{l}\text { Referência e Contrarreferência entre os serviços de } \\
\text { reabilitação da pessoa com deficiência: a (dess) } \\
\text { articulação na microrregião Centro-Sul Fluminense, RJ }\end{array}$ & BRASIL & ARTIGO \\
\hline 2018 & Aveionas et al & $\begin{array}{c}\text { Audielegy-Communication } \\
\text { Research }\end{array}$ & $\begin{array}{l}\text { Caracterização dos sistemas de referência e } \\
\text { contrarreferência em um serviço de alta complexidade na } \\
\text { cidade de São Paulo }\end{array}$ & BRASIL & ARTIGO \\
\hline
\end{tabular}

Fonte: Autores (2021).

Os estudos sobre a temática abrangeram estudos de abordagem quantitativa, qualitativa ou qualiquantitativos, e com objetos de estudo semelhantes sobre a organização dos fluxos de acesso aos serviços de saúde.

Dentre os estudos selecionados, destacam-se também que a busca por informações acerca das formas de organização da demanda na atenção secundária, modelos de gestão, acessibilidade e percepção do usuário são importantes para o planejamento em saúde (Vazquez et al., 2014).

\section{Discussão}

A regulação de encaminhamentos da APS para a atenção especializada é um problema ao mesmo tempo local e mundial. O Envelhecimento da população, avanços no conhecimento biomédico, aumento da prevalência das condições de saúde e critérios diagnósticos progressivamente mais estritos levaram a aumento das taxas de multimorbidade. Definir para cada protocolo as situações que devem e não devem ser encaminhadas para avaliação especializada, criando assim os gatilhos para encaminhamento da APS para a atenção especializada. Determinar quais situações apresentam maior risco e, portanto, devem ter acesso facilitado, considerando o melhor interesse do paciente. Em situações de tempos de espera prolongados, essas condições devem ter baixa prevalência.

Percebemos que o processo de organização dos fluxos de acesso aos serviços de saúde através de um sistema de referência e contrarreferência deve se consolidar para que a continuidade do cuidado do paciente seja contemplada. Esforços para melhorar o fluxo do paciente portanto, precisa incluir todas as partes do sistema, incluindo serviços ambulatoriais comunitários (Lewis et al., 2018). A qualidade do processo de referência pode ser avaliada em três dimensões: necessidade (se o paciente deve ser encaminhado), destino (onde o paciente deve ser referido) e qualidade (processo de encaminhamento, no qual a qualidade de encaminhamento é essencial) (Hartveit et al., 2017). 
Encaminhamentos adequados aumentam a qualidade do cuidado, apesar disso, a alta variabilidade dos encaminhamentos e ausência de parâmetros claros e taxas aceitáveis de encaminhamentos sugerem que tanto uso excessivo e deficiente da atenção especializada ocorrem. Além disso, a alta demanda por encaminhamentos aumenta a taxa de espera para consulta especializada (Molini-avejonas et al., 2018).

No processo de organização do Sistema Único de Saúde (SUS), a distribuição do serviço por meio de redes regionalizadas e a hierarquia da assistência tornam-se condições essenciais para melhorar a acessibilidade, viabilizando, assim, a aquisição dos serviços de saúde aos seus usuários (Molini-Avejonas et al., 2018).

O sistema de referência e contrarreferência constitui-se na articulação entre as unidades de saúde, sendo que por referência compreende-se o trânsito do nível menor para o de maior complexidade. Inversamente, a contrarreferência compreende o trânsito do nível de maior para o de menor complexidade. Alguns estudos realizados a partir de meados da década de 80 referem problemas na organização do referido Sistema, como a falta de informação por parte da população, problemas organizacionais intrínsecos aos próprios serviços e ainda problemas de integração e comunicação entre os vários níveis de atenção (Vazquez et al., 2014).

Destarte, as ações e serviços de saúde do SUS estão organizados de maneira regionalizada e hierarquizada em níveis crescentes de complexidade tecnológica, do primário e secundário ao nível terciário. Essa estruturação tem como objetivo alcançar a integralidade da assistência, entendida como um conjunto articulado e contínuo das ações e serviços preventivos e curativos, individuais e coletivos (Lewis et al., 2018)

A falta de integração entre diferentes pontos de atenção, a insuficiência de fluxos formais para atenção terciária e a desarticulação das políticas que normatizam a atenção secundária são entraves à garantia do cuidado integral, tornando incompleto esse processo na rede (Finkelsztejn et al., 2009).

Melhorar o acesso a cuidados especializados, o que inclui quaisquer serviços médicos especializados que só podem ser prestados por um médico especialista, foi identificada como um importante problema no nível do sistema de formigas, pois os resultados do paciente podem ser comprometidos quando o manejo da doença é adiado (Greenwood-lee et al., 2018).

"Melhorar o fluxo de pacientes através dos sistemas de saúde em um esforço para maximizar a capacidade e eficiência tem recebido atenção, mas a ênfase deste trabalho tem sido amplamente em ambientes de cuidados intensivos. Uma ampla gama de iniciativas tem sido usada para melhorar o fluxo do paciente e reduzir a espera tempos em ambientes ambulatoriais de cuidados de saúde, como: Avaliações para triagem, acesso avançado e racionamento (Pereira \& Machado, 2016)

A implementação da atenção secundária atualmente é um grande desafio para o alcance da integralidade no Sistema Único de Saúde (SUS), sendo necessário o desenvolvimento do fluxo de referência e contrarreferência, através do qual as informações e os usuários trafegam no sistema. As dificuldades encontradas nesse trajeto são definitivamente os entraves da integralidade. Superá-las e/ou amenizá-las, pode encurtar o caminho para a resolução das necessidades (Juliani; ciampone, 1999).

O sistema de encaminhamentos, chamado de "Referência e Contrarreferência" em nosso meio, faz parte de um dos atributos essenciais da APS, a coordenação única da atenção à saúde. Esta coordenação prevê um cuidado compartilhado entre o médico generalista e o especialista, de maneira a integrar as informações dos cuidados entre os diversos níveis do sistema de saúde (Erdmann et al., 2013).

O componente fundamental na integração dos níveis de saúde é o sistema efetivo de referência (origem) e contrarreferência (encaminhamento), no qual é realizado um fluxo de encaminhamentos entre os serviços, considerando-se seus diferentes níveis de complexidade. Reorganizar a lógica da necessidade técnica certa para cada usuário, em espaços e ocasiões adequados, em processos articulados de referência e contrarreferência, é uma prática e reflexão teórica desejável, tanto na formação, como na atuação dos diferentes profissionais de saúde (Katz et al., 2020) 
Nesse sentido citamos Erdmann et al., 2013 que relata que o aumento da resolubilidade na atenção primária depende do acesso a consultas e procedimentos disponíveis na atenção secundária. A boa relação entre a atenção primária e secundária é um dos fatores condicionantes dessa resolutividade, o que foi possível identificar no presente estudo. Investimentos em atenção secundária potencializam a resolubilidade da atenção primária. Por outro lado, a baixa resolubilidade da atenção primária aumenta a demanda para a atenção secundária.

A Atenção Primária à Saúde (APS), conforme denominação internacional, constitui o nível de atenção à saúde que se encontra mais próximo dos cidadãos e tem suas ações desenvolvidas nas unidades básicas de saúde (UBS). Ela representa a principal porta de entrada e centro de comunicação com toda a Rede de Atenção à Saúde, devendo se pautar nos princípios da universalidade, da acessibilidade, do vínculo, da continuidade do cuidado, da integralidade da atenção, da responsabilização, da humanização, da equidade e da participação social (Ministério da Saúde, 2012). Já aos níveis secundário e terciário, competem o planejamento e a execução de ações de média e alta complexidades, desenvolvidas nos ambulatórios de especialidades e em serviços hospitalares, normalmente utilizados em casos que requeiram atenção de profissionais especializados, recursos tecnológicos de maior densidade no apoio diagnóstico e terapêutico, não disponíveis na APS (Vazquez et al., 2014)

Contemplando a pergunta de revisão, salienta-se que há necessidade da construção de instrumentos consolidados para fluxos de acesso aos serviços de saúde, foi observado nos estudos a importância fundamental da integração dos três níveis de saúde com um sistema efetivo de referência e contrarreferência.

\section{Considerações Finais}

A organização dos fluxos de acesso aos serviços de saúde se torna uma tarefa inconclusiva, considerando que a Rede de Atenção não trabalha de forma integrada priorizando o seguimento e o cuidado centrado no paciente.

Precisa-se conhecer o fluxo de referência e contrarreferência de serviços de alta complexidade, bem como quem são os usuários, suas necessidades e grau de satisfação com os serviços, é imprescindível na soma de esforços para aperfeiçoamento da assistência das unidades de alta complexidade.

Os resultados desta revisão demonstram que a acessibilidade é um desafio ubíquo e deficiências na porta de entrada prejudicam a consecução dos demais atributos essenciais da Atenção Primária à Saúde.

\section{Referências}

Bardin, L. (2011) Análise de conteúdo. Edições, 70 p.

Base de dados (2021). http://www2.eca.usp.br/prof/sueli/cbd201/bases.htm.

Botelho, L. L. R., Cunha, C. C. A. \& Macedo, M. (2011). O método da revisão integrativa nos estudos organizacionais. Gestão e Sociedade. 5(11), 12136. https://doi.org/10.21171/ges.v5i11.1220.

Clarivate. (2021). EndNote Web. Website da Clarivate. https://access.clarivate.com/login?app=endnote".

Estrela, C. (2018). Metodologia Científica: Ciência, Ensino, Pesquisa. Editora Artes Médicas.". Autores, coloquem em itálico o nome do livro.

Erdmann, A. L. et al. (2013). A atenção secundária em saúde: Melhores práticas na rede de serviços. Revista Latino-Americana de Enfermagem, 21, 131-139.

Florêncio, R. S., Moreira, T. M. M., Pessoa, V. L. M. de P., Cestari, V. R. F., Silva, V. M. G. N., Rabelo, S. M. S., Pereira, M. L. D., Santiago, J. C. dos S., Borges, J. W. P., Mattos, S. M., Silva, M. R. F. da, \& Ribeiro, D. C. (2020). Mapeamento dos estudos sobre vulnerabilidade em saúde: uma revisão de escopo. Research, Society and Development, 9(10), e2079108393.

Finkelsztejn, A. et al. (2009). Encaminhamentos da atenção primária para avaliação neurológica em Porto Alegre, Brasil. Physis: Revista de Saúde Coletiva. 19(3), 731-41.

Greenwood-lee, J. et al. (2018). A categorisation of problems and solutions to improve patient referrals from primary to specialty care. BMC Health Services Research, 18(1), 1-16. 
Research, Society and Development, v. 11, n. 1, e16011124574, 2022

(CC BY 4.0) | ISSN 2525-3409 | DOI: http://dx.doi.org/10.33448/rsd-v11i1.24574

Hartveit, M. et al. (2017). Quality indicators for the referral process from primary to specialised mental health care: an explorative study in accordance with the RAND appropriateness method. BMC Health Services Research, 17(1), 1-13.

Juliani, C. M., \& Ciampone, M. H. (1999). Counter-reference and reference system organization in the health system context: perception of nurses. Revista da Escola de Enfermagem da USP, 33(4), 323-333.

Katz, N. et al (2020). Access and regulation of specialized care in Rio Grande do Sul: The regulaSUS strategy of telessaúdeRS-UFRGS. Ciencia e Saude Coletiva, 25(4), 1389-1400

Lewis, A. K. et al.(2018). Reducing wait time from referral to first visit for community outpatient services may contribute to better health outcomes: A systematic review. BMC Health Services Research, 18(1), 1-14, 2018.

Molini-avejonas, D. R. et al. (2018). Caracterização dos sistemas de referência e contrarreferência em um serviço de fonoaudiologia de alta complexidade na cidade de São Paulo. Audiology - Communication Research, 23, 1-10.

Ministério da Saúde. (2010). Portaria no 4.279, de 30 de dezembro de 2010. Estabelece diretrizes para a organização da Rede de Atenção à Saúde no âmbito do Sistema Único de Saúde - SUS. Brasília.

Mendes, Eugênio Vilaça. (2019). Desafios do SUS. CONASS, 869 p).

Mendes, K. D. S., Silveira, R. C. P. S. \& Galvão, C. M. (2008). Revisão Integrativa: método de pesquisa para a incorporação de evidências na saúde e na enfermagem. Texto Contexto Enfer. 17(4).

Pereira, J. S., \& Machado, W. C. A. (2016). Referência e contrarreferência entre os serviços de reabilitação física da pessoa com deficiência: a (des) articulação na microrregião Centro-Sul Fluminense, Rio de Janeiro, Brasil. Physis: Revista de Saúde Coletiva, 26(3), 1033-1051.

Vazquez, F. L. et al. (2014). Referência e Contrarreferência na Atenção Secundária em Odontologia em Campinas, SP, Brasil. Ciencia e Saude Coletiva, 19(1), 245-255. 